\title{
Cyclic Convolution Operators on the Hardy Spaces
}

\author{
K. Hedayatian M. Faghih-Ahmadi
}

\begin{abstract}
Using Banach algebra structure of the Hardy space, we describe all finite codimensional invariant subspaces of a cyclic convolution operator on the Hardy space $H^{p}$ of the unit disc for $1 \leq p \leq \infty$. We also observe that every operator in the commutant of such operators is not weakly supercyclic.
\end{abstract}

\section{Introduction}

Let $X$ be a Banach space and $T$ be a bounded linear operator on $X$. A subspace $M$ of $X$ is an invariant subspace for $T$, if $T M \subseteq M$; if further, $\operatorname{dim} X / M<\infty$, it is called a finite codimensional invariant subspace for $T$. The famous "invariant subspace problem" asks whether every bounded linear operator on a Hilbert space, or generally, on a Banach space, admits a non-trivial invariant subspace. Of course, the problem has been answered, negatively, by Read [13] for the Banach space setting. A more specific problem, in this connection, is the characterization of all invariant subspaces of an operator. Among bounded operators, the classes of shift operators, multiplication operators, and integral operators have a special position. The invariant subspaces of Volterra integral operator on the Hilbert Hardy space is studied by Donoghue [8], and a complete characterization of such subspaces in a Banach spaces of analytic functions in the open unit disc, containing the Hardy, Bergman and Dirichlet spaces is obtained in 2008 by Aleman and Korenblum in [4]. Furthermore, characterizing finite codimensional

Received by the editors in July 2014 - In revised form in December 2014.

Communicated by F. Bastin.

2010 Mathematics Subject Classification : 47A16, 47B38, 46E10.

Key words and phrases : cyclic convolution operators, Hardy spaces. 
invariant subspaces of operators, specially on spaces of analytic functions, is considered by several authors. In 1969, Gellar determined the finite codimensional invariant subspaces of weighted shifts [10]. Axler and Bourdon [6] and also Aleman [2] described these subspaces for the operator multiplication by $z$ on the Bergman spaces. Later, Aleman [3] generalized their results to a Hilbert space of analytic functions. Abdollahi and Seddighi, in 1999, investigated this problem on a Banach space of analytic functions [1]. Moreover, Carisson, in 2011, obtained a characterization for a finite codimensional invariant subspaces of multiplication by a polynomial in Banach spaces of vector-valued analytic functions in several variables [7].

Supercyclicity of operators is also a concept closely related to the invariant subspace problem. It was first introduced by Hilden and Wallen in [11]. Recall that an operator $T$ is (weakly) supercyclic if the set of all scalar multiples of the vectors in $\left\{x, T x, T^{2} x, \cdots\right\}$ is (weakly) dense in $X$. In this case, the vector $x$ is called a (weakly) supercyclic vector for $T$. Sanders proved that there exists a weakly supercyclic operator that fail to be norm supercyclic [14]. Sufficient conditions for non-weak supercyclicity of a weighted composition operator on a Hilbert space of analytic functions are given in [12]. Non-supercyclicity of the Volterra operator on $L^{p}[0,1], 1 \leq p<\infty$ is proved in [9]. Later, Shkarin [16] generalized this result, and proved that operators commuting with the Volterra operator on such spaces are not weakly supercyclic. In this paper, we are going to prove that operators in the commutant of a cyclic convolution operator on the Hardy space are not weakly supercyclic.

This paper is organized as follows. After presenting some preliminaries in Section 2, we are going to characterize all finite codimensional invariant subspaces of a cyclic convolution operator, in Section 3. The paper proceeds, in Section 4, with the study of weak supercyclicity of the operators commuting with a cyclic convolution operator; indeed, we prove that they are not weakly supercyclic.

\section{Preliminary Results}

Throughout this paper, the open unit disc is denoted by $\mathbb{D}$ and $\partial \mathbb{D}$ is its boundary. Also, $H(\mathbb{D})$ is the set of all analytic functions on $\mathbb{D}$. To a function $f$ in $H(\mathbb{D})$, we associate a family of functions $\left\{f_{r}\right\}_{r}$ on $\partial \mathbb{D}$ defined by

$$
f_{r}\left(e^{i \theta}\right)=f\left(r e^{i \theta}\right) \quad(0 \leq r<1) .
$$

Let $m$ denote the normalized Lebesgue measure on $\partial \mathbb{D}\left(\right.$ i.e., $\left.d m=\frac{d \theta}{2 \pi}\right)$. By the $p$-norm of $f_{r}$ we mean

$$
\begin{aligned}
& \left\|f_{r}\right\|_{p}=\left(\int_{\partial \mathbb{D}}\left|f_{r}\right|^{p} d m\right)^{\frac{1}{p}} \quad(1 \leq p<\infty) ; \\
& \left\|f_{r}\right\|_{\infty}=\sup _{0 \leq \theta<2 \pi}\left|f\left(r e^{i \theta}\right)\right| .
\end{aligned}
$$

Also, we define the $p$-norm of $f$ by

$$
\|f\|_{p}=\sup \left\{\left\|f_{r}\right\|_{p}: 0 \leq r<1\right\} \quad(1 \leq p \leq \infty) .
$$


The Hardy space $H^{p}(1 \leq p \leq \infty)$ is the class of all analytic functions $f$ on $\mathbb{D}$, for which $\|f\|_{p}<\infty$. The norm $\|.\|_{p}$ turns $H^{p}$ into a Banach space. It shall be easily verified that if $1 \leq q \leq p \leq \infty$, then

$$
H^{\infty} \subseteq H^{p} \subseteq H^{q}
$$

Furthermore, the functional $f \mapsto f^{(n)}(z)$ is continuous on $H^{p}$, for every $z \in \mathbb{D}$ and $n \geq 0$.

Recall that the space $H^{p}, 1 \leq p \leq \infty$ with the product

$$
\begin{aligned}
(f * g)(z) & =\frac{d}{d z} \int_{0}^{z} f(z-t) g(t) d t \\
& =\int_{0}^{z} f^{\prime}(z-t) g(t) d t+f(0) g(z)
\end{aligned}
$$

becomes a commutative Banach algebra with the constant function 1 as the identity. Indeed, there exists a constant $C_{p}$ depending only on $p$ such that $\|f * g\|_{p} \leq$ $C_{p}\|f\|_{p}\|g\|_{p}$. Moreover, $f \in H^{p}$ is invertible if and only if $f(0) \neq 0$ (see [17]).

For $\varphi \in H^{\infty}$ define the multiplication operator $M_{\varphi}$ on $H^{p}$ by $M_{\varphi} f=\varphi f$. It is known that $\left\|M_{\varphi}\right\| \leq\|\varphi\|_{\infty}$. Moreover, for $f \in H^{p}$ the multiplication operator with $*$-product on $H^{p}$ is denoted by $D_{f}$ which is given by $D_{f} g=f * g$. The operator $D_{f}$ is bounded; indeed,

$$
\left\|D_{f}\right\| \leq C_{p}\|f\|_{p}
$$

Let $1 \leq p \leq \infty$ and $w \in H^{p}$. The convolution operator $K_{w}$ on $H^{p}$ is defined by

$$
\left(K_{w} g\right)(z)=\int_{0}^{z} w(z-t) g(t) d t .
$$

When $w$ is the constant function 1 , the operator $K_{w}$ is the Volterra integral operator which is denoted by $\mathrm{V}$.

\section{Finite Codimensional Invariant Subspaces}

It is known that the Volterra integral operator is a compact, quasinilpotent operator [5]. The following result generalizes this fact.

Proposition 1. Let $1 \leq p \leq \infty$ and $w \in H^{p}$. Then the convolution operator $K_{w}$ is a bounded, compact (for $p \neq \infty$ ) and quasinilpotent operator on $\mathrm{H}^{p}$.

Proof. Suppose that $g \in H^{p}$ and $z \in \mathbb{D}$. Then

$$
\begin{gathered}
\left(D_{V(w)} g\right)(z)=(V(w) * g)(z)=\int_{0}^{z}(V(w))^{\prime}(z-t) g(t) d t \\
=\int_{0}^{z} w(z-t) g(t) d t=\left(K_{w} g\right)(z) .
\end{gathered}
$$

Consequently, $K_{w}=D_{V(w)}$ is a bounded operator. It is shown in [5] that for any polynomial $q$ the operator

$$
T_{q}(f)(z)=\frac{1}{z} \int_{0}^{z} q(t) f(t) d t
$$


is a compact operator on $H^{p},(1 \leq p<\infty)$. Thus, the operator

$$
S_{q}(f)=\frac{1}{z} \int_{0}^{z} q(z-t) f(t) d t
$$

is compact. Hence for $\varphi(z)=z$, the operator $K_{q}=M_{\varphi} S_{q}$ is also compact. Now, let $\left(q_{n}\right)_{n}$ be a sequence of polynomials converging to $w$ in $H^{p}$. Since

$$
\begin{gathered}
\left\|K_{q_{n}}-K_{w}\right\|=\left\|D_{V\left(q_{n}\right)-V(w)}\right\| \\
\leq C_{p}\left\|V\left(q_{n}-w\right)\right\| \leq C_{p}\|V\|\left\|q_{n}-w\right\|,
\end{gathered}
$$

we conclude that $K_{w}$ is a compact operator.

Note that $f \in H^{p}$ is invertible (as an element of a Banach algebra) if and only if $f(0) \neq 0$. Thus, the spectrum of $f$ is $\sigma(f)=\{f(0)\}$. This, in turn, implies that

$$
\begin{gathered}
\sigma\left(K_{w}\right)=\sigma\left(D_{V(w)}\right)=\sigma(V(w)) \\
=\{(V(w))(0)\}=\{0\} .
\end{gathered}
$$

Hence $K_{w}$ is a quasinilpotent operator.

In the sequel, we assume that $1 \leq p \leq \infty$. Recall that if $X$ is a Banach space and $T$ is a bounded linear operator on $X$ then $x \in X$ is called a cyclic vector for $T$ whenever $\bigvee\left\{T^{n} x: n \geq 0\right\}=X$; here $\bigvee\{$.$\} denotes the closed linear span of the$ set $\{$.$\} . Also, if T$ has a cyclic vector then it is called a cyclic operator.

Theorem 1. Suppose that the convolution operator $K_{w}$ is cyclic on $H^{p}$. Then a vector $f \in H^{p}$ is a cyclic vector for $K_{w}$ if and only if $f(0) \neq 0$.

Proof. Take a cyclic vector $f \in H^{p}$ for $K_{w}$. So there is a sequence of polynomials $\left(P_{n}\right)_{n}$ such that $P_{n}\left(K_{w}\right) f \rightarrow 1$ in $H^{p}$. Consequently,

$$
\left(P_{n}\left(K_{w}\right) f\right)(0)=P_{n}(0) f(0) \rightarrow 1,
$$

which implies that $f(0) \neq 0$. Thus, the operator $D_{f}$ is invertible. On the other hand, since $H^{p}$ is a commutative Banach algebra

$$
K_{w} D_{f}=D_{f} K_{w}
$$

and so

$$
D_{f}^{-1} K_{w}=K_{w} D_{f}^{-1} .
$$

Since $f$ is a cyclic vector for $K_{w}$, the above equality shows that the constant function 1 is a cyclic vector for $K_{w}$.

Now, let $f$ be an arbitrary element in $H^{p}$ such that $f(0) \neq 0$. Since the constant function 1 is a cyclic vector for $K_{w}$ we conclude from (1) that $f$ is a cyclic vector of $K_{w}$.

Corollary 1. A vector $f \in H^{p}$ is a cyclic vector for the Volterra operator $V$ if and only if $f(0) \neq 0$. 
Proof. Since $V^{n} 1=z^{n} / n$ ! for every $n \geq 0$, the operator $V$ is cyclic. So the result follows from Theorem 1 .

In what follows, we use the notation $f^{* n}=\underbrace{f * f * \cdots * f}_{n \text {-times }}$ for every $n \geq 1$ and every $f \in H^{p}$.

Proposition 2. If $K_{w}$ is cyclic then for every $n \geq 0$,

$$
\overline{z^{* n} * H^{p}}=\bigvee\left\{z^{n}, K_{w} z^{n}, K_{w}^{2} z^{n}, \cdots\right\}=z^{n} H^{p} .
$$

Proof. By Theorem 1, the constant function 1 is a cyclic vector for $K_{w}$; so the above equality holds for $n=0$. Suppose that $n \geq 1$ and let $Q_{n}$ be the set of all polynomials $q$ so that

$$
q(0)=q^{\prime}(0)=\cdots=q^{(n-1)}(0)=0 .
$$

Since $z^{* n} * q^{(n)}=q$ for every polynomial $q$ and $Q_{n}$ is dense in $z^{n} H^{p}$ we conclude that $z^{n} H^{p} \subseteq \overline{z^{* n} * H^{p}}$. Moreover, for $f \in H^{p}$ there is a sequence of polynomials $\left(q_{i}\right)_{i}$ such that

$$
q_{i}\left(K_{w}\right) z^{n}=D_{z^{n}} q_{i}\left(K_{w}\right) 1 \rightarrow D_{z^{n}} f
$$

as $i \rightarrow \infty$. Therefore,

$$
z^{n} H^{p} \subseteq \overline{z^{* n} * H^{p}} \subseteq \bigvee\left\{z^{n}, K_{w} z^{n}, K_{w}^{2} z^{n}, \cdots\right\}
$$

On the other hand, it is easy to see that

$$
(f * g)^{(k)}(z)=\int_{0}^{z} f^{(k)}(z-t) g^{\prime}(t) d t+\sum_{i=0}^{k-1} f^{(i)}(0) g^{(k-i)}(z)+g(0) f^{(k)}(z)
$$

for all $f$ and $g$ in $H^{p}$. Put $f(z)=z^{n}$. So $\left(z^{n} * g\right)^{(k)}(0)=0$ for all $g \in H^{p}$ and $0 \leq k \leq n-1$. Therefore, for every $i \geq 0$ and $0 \leq k \leq n-1$, we have

$$
\left(K_{w}^{i} z^{n}\right)^{(k)}(0)=\left((V w)^{* i} * z^{n}\right)^{(k)}(0)=0 .
$$

Consequently,

$$
\bigvee\left\{z^{n}, K_{w} z^{n}, K_{w}^{2} z^{n}, \cdots\right\} \subseteq z^{n} H^{p}
$$

Hence

$$
\overline{z^{* n} * H^{p}}=\bigvee\left\{z^{n}, K_{w} z^{n}, K_{w}^{2} z^{n}, \cdots\right\}=z^{n} H^{p} .
$$

It is known [4] that for every nonzero invariant subspace $M$ of the Volterra operator $V$ on the space $H^{p}, p>1$ there is a nonnegative integer $n$ such that $M=z^{n} H^{p}$. In the next theorem, we characterize all finite codimensional invariant subspaces of a cyclic convolution operator on $H^{p}$.

Theorem 2. Suppose that $K_{w}$ is cyclic on $H^{p}$. Then the following results hold.

(a) Every proper invariant subspace of $K_{w}$ is a subset of $z \mathrm{H}^{p}$.

(b) Every invariant subspace of $K_{w}$ of finite codimension, has the form $\overline{K_{w}^{n} H^{p}}$ for some $n \geq 0$. 
Proof. (a) Suppose that $M$ is a proper invariant subspace of $K_{w}$ and $f \in M$. If $f(0) \neq 0$ then by Theorem 1 , the vector $f$ is a cyclic vector of $K_{w}$, and so $M=H^{p}$; which is a contradiction. Hence $M \subseteq z H^{p}$.

(b) Let $M$ be an invariant subspace of $K_{w}$ such that $\operatorname{dim} H^{p} / M=m \geq 0$. Define the linear transformation $T: H^{p} / M \rightarrow H^{p} / M$ by $T(f+M)=K_{w} f+M$, which is well defined thanks to the fact that $M$ is an invariant subspace for $K_{w}$. Let $q(z)$ be the characteristic polynomial of $T$. Then $q(z)$ is a monic polynomial of degree $m$ such that, by the Cayley-Hamilton theorem, $q(T)=0$.

Suppose $n \geq 0$ is the multiplicity of the zero of $q(z)$ at the origin. Thus $q(z)=$ $z^{n} t(z)$ where $t(z)$ is a polynomial whose roots are nonzero. Now, if $\lambda \neq 0$ is a root of $t(z)$, then $K_{w}-\lambda$ is invertible. Indeed, $\sigma\left(K_{w}-\lambda\right)=\{\lambda\}$ therefore, $t\left(K_{w}\right)$ is invertible and so

$$
K_{w}^{n} H^{p}=K_{w}^{n} t\left(K_{w}\right) H^{p}=q\left(K_{w}\right) H^{p} \subseteq M .
$$

The last inclusion occurs because $q(T)=0$. On the other hand, since $K_{w}$ is cyclic

$$
\operatorname{dim} H^{p} / M \leq \operatorname{dim} H^{p} / \overline{K_{w}^{n} H^{p}} \leq n \leq m=\operatorname{dim} H^{p} / M .
$$

Hence $M=\overline{K_{w}^{n} H^{p}}$.

Remark. In the above proof, although $q(T)=0$, but $q\left(K_{w}\right)$ is never zero. Because otherwise, (2) shows that $K_{w}$ is a nilpotent operator. But this is impossible, thanks to the fact that the constant function 1 is a cyclic vector for $K_{w}$.

\section{Non-weak Supercyclicity of the Commutant}

Recall that for an operator $T$ on a Banach space $X$ the commutant of $T$, which is denoted by $\{T\}^{\prime}$, is the set of all bounded operators $S$ on $X$ such that $T S=S T$. First, we obtain the commutant of a cyclic convolution operator on the Hardy space.

Proposition 3. Suppose that $K_{w}$ is cyclic on the space $H^{p}$. Then $A \in\left\{K_{w}\right\}^{\prime}$ if and only if $A=D_{f}$ where $f=A 1$.

Proof. Suppose that $A$ commutes with $K_{w}$. Since $K_{w}=D_{V(w)}, A D_{V(w)}^{n}=D_{V(w)}^{n} A$ for all $n \geq 0$. Hence

$$
A\left(V(w)^{* n}\right)=A D_{V(w)}^{n} 1=D_{V(w)}^{n} A 1=D_{f}\left(V(w)^{* n}\right)
$$

where $f=A 1$.

Since the constant function 1 is a cyclic vector for $K_{w}, \bigvee\left\{1, V(w), V^{* 2}(w)\right.$, $\cdots\}=H^{p}$. Consequently, $A=D_{f}$. The reverse inclusion is obvious. 
Theorem 3. Every operator in the commutant of a cyclic convolution operator $K_{w}$ on $H^{p}$ is not weakly supercyclic.

Proof. Suppose that $T \in\left\{K_{w}\right\}^{\prime}$. By the previous proposition $T=D_{h}$ for some $h \in H^{p}$. If $h(0)=0$ then $\left(D_{h}^{n} g\right)(0)=0$ for every $n \geq 0$ and every $g \in H^{p}$. Hence $T$ cannot be a weakly supercyclic operator. Thus, we may assume that $h(0) \neq 0$. Now consider the bounded linear functional $\Lambda$ on $H^{p}$ defined by $\Lambda(f)=f(0)$. Observe that $\operatorname{ker} \Lambda$ is a closed invariant subspace of $T$ of codimension 1. Define the bounded linear operator $S$ on $\operatorname{ker} \Lambda \oplus \mathbb{C}$ by

$$
S(f \oplus \lambda)=\frac{1}{h(0)}\left(\left.T\right|_{\operatorname{ker} \Lambda} f+\lambda(h-h(0))\right) \oplus \lambda
$$

and consider the bounded linear bijective transformation $A: \operatorname{ker} \Lambda \oplus \mathbb{C} \rightarrow H^{p}$ by $A(f \oplus \lambda)=f+\lambda$.

Now for every $f \in H^{p}$, the fact that $D_{h}(f(0))=f(0) h$ implies that

$$
\begin{aligned}
\left(A S A^{-1}\right)(f) & =(A S)((f-f(0)) \oplus f(0)) \\
& =A\left(\frac{1}{h(0)}\left(D_{h}(f-f(0))+f(0)(h-h(0))\right) \oplus f(0)\right) \\
& =\frac{1}{h(0)}\left(D_{h}(f-f(0))+D_{h}(f(0))\right) \\
& =\frac{1}{h(0)} T f .
\end{aligned}
$$

Assume, on the contrary that, the operator $T$ is weakly supercyclic. Since similarity preserves weak supercyclicity, we conclude that the operator $S$ is also weakly supercyclic. Thus, Corollary 7.11 and Lemma 7.12 of [15] imply that $B:=\left.T\right|_{\operatorname{ker} \Lambda}$ is weakly hypercyclic; i.e., there is a vector $g \in \operatorname{ker} \Lambda$ such that the set $\left\{g, B g B^{2} g, \cdots\right\}$ is weakly dense in $\operatorname{ker} \Lambda$. Then, for every $f \in \operatorname{ker} \Lambda$ there is a net $\left(n_{\alpha}\right)_{\alpha}$ such that $B^{n_{\alpha}} g=D_{h}^{n_{\alpha}} g=h^{* n_{\alpha}} * g$ converges weakly to $f$. Furthermore, in view of the continuity of the linear functional $f \mapsto f^{\prime}(0)$, we see that

$$
\left(h^{* n_{\alpha}} * g\right)^{\prime}(0)=h^{* n_{\alpha}}(0) g^{\prime}(0)=h^{n_{\alpha}}(0) g^{\prime}(0) \rightarrow f^{\prime}(0) .
$$

Taking $f(z)=z$ in the above formula, we see that $g^{\prime}(0) \neq 0$ and $|h(0)|=1$. But when $f(z)=z^{2}$ we get a contradiction.

Acknowledgments. This research was in part supported by a grant from Shiraz University Research Council.

\section{References}

[1] A. Abdollahi and K. Seddighi, Finite codimensional invariant subspaces of Banach spaces of analytic functions, Rocky Mountain J. Math. 29(1999), 369-381.

[2] A. Aleman, Invariant subspaces with finite codimension in Bergman spaces, Trans. Amer. Math. Soc. 330(1992), 531-544. 
[3] A. Aleman, Finite codimensional invariant subspaces of Hilbert spaces of analytic functions, J. Funct. Anal. 119(1994), 1-18.

[4] A. Aleman, B. Korenblum, Volterra invariant subspaces of $H^{p}$, Bull. Sci. Math. 132 (2008), 510-528.

[5] A. Aleman, A. G. Siskakis, An integral operator on $H^{p}$, Complex Variable Theory Appal. 28 (1995), 149-158.

[6] S. Axler and P. Bourdon, Finite codimension invariant subspaces of Bergman spaces, Trans. Amer. Math. Soc. 306(1988), 805-817.

[7] M. Carisson, Invariant subspaces of finite codimension in Banach spaces of analytic functions, J. Math. Anal. Appl. 373 (2011), 1-12.

[8] W. F. Donoghue, The lattice of invariant subspaces of a completely continuous quasinilpotent transformation, Pacif. J. Math. 7 (1957), 1031-1035.

[9] E. Gallardo-Gutiérrez and A. Montes-Rodríguez, The Volterra operator is not supercyclic, Integr. Equ. Oper. Theory 50 (2004), 211-216.

[10] R. Gellar, Cyclic vectors and parts of the spectrum of a weighted shift, Trans. Amer. Math. Soc. 146(1969), 69-85.

[11] H. Hilden and L. Wallen, Some cyclic and non-cyclic vectors of certain operators, Indiana Math. J. 23 (1973), 557-565.

[12] Z. Kamali, K. Hedayatian, and B. Khani Robati, Non-weakly supercyclic weighted composition operators, Abstract and Applied Analysis, Volume 2010, Article ID 143808, 14 pages.

[13] C. J. Read, The invariant subspace problem for a class of Banach spaces, II. Hypercyclic operators. Israel J. Math., 63 (1998), 1-40.

[14] R. Sanders, Weakly supercyclic operators, J. Math. Anal. Appl. 292 (2004), 148-159.

[15] S. Shkarin, Universal elements for non-linear operators and their applications, J. Math. Anal. Appl. 348 (2008), 193-210.

[16] S. Shkarin, Operators commuting with the Volterra operator are not weakly supercyclic, Integr. Equ. Oper. Theory 68 (2010), 229 -241.

[17] N. Wigley, A Banach algebra structure for $H^{p}$, Canad. Math. Bull., 18 (1975), 597-603.

Department of Mathematics, College of Sciences, Shiraz University, Shiraz 71454, Iran

Email addresses: hedayati@shirazu.ac.ir (khedayatian@gmail.com), faghiha@shirazu.ac.ir 